\title{
DU TOURISME GÉNÉALOGIQUE DANS L'IRLANDE CONTEMPORAINE
}

\author{
Caroline LEGRAND
}

RÉSUMÉ : À l'aide de données anthropologiques contemporaines, cet article entend explorer les raisons d'une expansion surprenante et toute récente d'un tourisme mémoriel en Irlande. En effet, de nombreux descendants de migrants irlandais « reviennent» aujourd'hui découvrir la terre de leurs ancêtres. S'intéressant à des formes particulières de «retour au pays » que sont les rassemblements claniques, l'auteur souligne que ces manifestations collectives et ritualisées revêtent une double dimension. Outre leur enjeu identitaire, elles constituent aussi un moyen pour les gouvernements irlandais et nord-irlandais de développer l'activité touristique sur l'Île tout entière et d'en diversifier les ressources économiques.

MoTS-CLÉS : migration, généalogie, mémoire, Irlande, tourisme, rassemblements claniques.

ABSTRACT : The aim of this article is to explore the causes of the recent and surprising expansion of heritage tourism in Ireland, working with contemporary anthropological data. It is a fact that numerous descendants of Irish migrants have been returning 'home' recently to discover the land where their forebears originally came from. Of particular interest here are specific types of return to the homeland, such as the gathering of clans. We would like to point out here that such collective, ritualized activities have a twofold nature: in addition to their role in the forging of identity, they provide a means for the governments of Ireland and Northern Ireland to develop touristic activities over the entire island, thus diversifying economic resources.

KEYWORDS : migration, genealogy, memory, Ireland, tourism, clan gatherings. 
ZuSAMmENFASSUnG : Mit Hilfe zeitgenössischer anthropologischer Daten möchte dieser Artikel die Gründe des sehr neuen und überraschenden «Gedenk »-Tourismus nach Irland erforschen. Tatsächlich kehren viele Nachkommen irischer Migranten heute wieder ins Land ihrer Vorfahren "zurück». Der Autor interessiert sich für die besonderen Formen der "Heimkehr», nämlich die Zusammenkünfte der Klanns und hebt die doppelte Dimension dieser kollektiven und ritualisierten Veranstaltungen hervor. Sie spielen nicht nur eine Rolle bei der Identitätsfindung, sondern ermöglichen ausserdem der irischen und nordirischen Regierung den Tourismus als solchen auf der ganzen Insel auszubauen und über vielseitigere wirtschaftliche Mittel zu verfügen.

STICHWÖRTER : Auswanderung, Genealogie, Gedächtnis, Irland, Tourismus, klanische Zusammenkünfte.

Caroline LEGRAND, née en 1973, est doctorante à l'École des hautes études en sciences sociales. Elle y prépare actuellement une thèse d'anthropologie sociale sur les représentations et usages sociaux des généalogies irlandaises à l'époque contemporaine, sous la direction de Françoise Zonabend. Elle est rattachée au laboratoire d'Anthropologie sociale et est membre de la Société française d'études irlandaises. Parallèlement à sa recherche doctorale, elle a participé à une étude transversale menée sur les usagers de l'Aide sociale à l'enfance de Seine-Saint-Denis, puis enseigné en tant qu'ATER l'ethnologie à l'université de Bretagne occidentale (Brest).

Adresse: École des hautes études en sciences sociales, laboratoire d'Anthropologie sociale, 52 rue du Cardinal-Lemoine, F-75005 Paris.

Courrier électronique : carolinelegrand@voila.fr 


\begin{abstract}
«Le monde entier essaie de découvrir ses racines, mais alors que la plupart sont contraints de chercher à tâtons ; nous avons, nous ici, un avantage unique. Nous pouvons retracer notre filiation sur plus de cinq mille ans, et cela est d’un intérêt phénoménal. »
\end{abstract}

Charles HaUgheY ${ }^{1}$

En 1999, une journaliste titrait son article paru dans un hebdomadaire irlandais « Back to their roots ${ }^{2}$ », faisant référence aux milliers de descendants de migrants irlandais venant chaque année consulter les archives entreposées à la Bibliothèque nationale pour y découvrir des informations biographiques concernant leurs ancêtres ; tandis que d'autres éditorialistes relataient dans la presse nationale ou régionale les événements particuliers auxquels prenaient part ces mêmes individus sur le sol irlandais dans l'espoir d'y rencontrer d'autres « historiens de famille ${ }^{3} »$. Cette forme particulière de tourisme qualifiée en Irlande de « généalogique »- on y parle de «roots-tourism »-s'est massivement développée au cours de ces vingt dernières années. Plusieurs éléments sont à l'origine de son expansion. Tout d'abord, l'histoire même de ce pays et l'importance des vagues d'émigration qui s'y sont succédé 4 font que de très

1. Cité par Moyra Kneafsey, in KocKEL, éd., 1995, p. 147. Ici et dans la suite de cet article, les traductions des textes anglais en français ont été effectuées par Steven Schaeffer et revus par l'auteur.

2. B. O'Hanlon, « Back to their roots », Sunday Tribune, 18 juil. 1999.

3. L'utilisation dans cet article de cette expression ne renvoie pas aux scientifiques qui travaillent dans une perspective historique sur $l a$ famille irlandaise, mais à ceux qui, comme les généalogistes - professionnels ou amateurs -, tentent de reconstruire l'histoire de leur famille et se désignent de ce fait comme des « historiens de famille» (Family historians).

4. Située en bordure occidentale de l'Europe, la république d'Irlande compte aujourd'hui près de 3,5 millions d'habitants contre plus de 8 millions au début du XIX ${ }^{\mathrm{e}}$ siècle. Les différentes vagues d'émigration qui touchèrent le pays au cours de son histoire sont considérées à juste titre, par les historiens et démographes, comme les principales responsables de sa dépopulation. Remontant bien au-delà de la Grande Famine, comme l'indiquent VerRIÈRE, 1979, et DUPÂQUIER, 1980, ces mouvements internationaux de populations trouvent leurs origines dans le contexte religieux, politique, économique de l'Île, et ne s'estompèrent qu'à partir du moment où l'Irlande parvint à fournir à ses habitants les moyens d'y prospérer localement, ce que montre Hannan, 1979. Aujourd'hui, avec un taux de croissance économique de plus de 7\% entre 1995 et 1999, l'Irlande attire plus qu'elle ne refoule et nombreux sont ceux à s'y installer à titre provisoire ou définitif, comme l'expliquent MACÉinRí, 1997, et Catherine Piola, in Gallagher, dir., 1998, p. 61-70 ; le solde migratoire de l'île étant d'ailleurs récemment devenu positif. 
nombreuses personnes peuvent à travers le monde revendiquer une ascendance irlandaise. La volonté qu'ont certaines d'entre elles de mieux connaître leur passé familial et la terre sur laquelle ont vécu leurs aïeuls peut expliquer l'engouement que suscite en Irlande l'activité généalogique. En cela, les séjours des descendants de migrants en Irlande revêtent un enjeu identitaire. Mais ils sont aussi une source potentielle de bénéfice économique pour le gouvernement irlandais. L'observation ethnographique de ces mouvements de populations et des pratiques sociales auxquelles elles donnent lieu aujourd'hui ${ }^{5}$ permettront enfin de s'interroger sur les représentations qui lui sont associées : celles d'un espace et d'une identité.

Parler de « retour» pour désigner les déplacements qu'effectuent les descendants de migrants sur la terre de leurs ancêtres peut sembler impropre dans la mesure où, le plus souvent, ce ne sont pas ces voyageurs qui sont partis d'Irlande, mais leurs aïeuls. Malgré le décalage générationnel qui existe entre ceux qui ont quitté l'Irlande hier et ceux qui s'y rendent aujourd'hui, c'est pourtant ce terme que choisissent d'utiliser les descendants de migrants et les autochtones irlandais pour désigner de tels mouvements de population «Homecoming », «Housecoming ${ }^{6}$. De manière générale, les déplacements de descendants de migrants irlandais entre un espace vécu et celui de leurs ancêtres n'ont jusqu'à présent que peu retenu l'attention des chercheurs. George Gmelch est, semble-t-il, l'un des seuls anthropologues à s'y être intéressé en tentant d'établir une corrélation entre les motifs énoncés par les descendants de migrants pour justifier leurs déplacements et les lieux où ces derniers se rendaient. Il en a ainsi conclu que ceux qui se rendaient dans la campagne irlandaise étaient généralement motivés par des questions d'ordre identitaire, tandis que ceux qui allaient à Dublin ou dans d'autres grandes villes le faisaient souvent pour des raisons professionnelles ${ }^{7}$. Et si plusieurs chercheurs ont fait depuis mention d'une pratique de circulation étroitement liée avec un exercice « généalogique ${ }^{8}$ », aucun travail ne lui a été à ce jour spécifiquement consacré.

5. Ces données ont été recueillies dans le comté de Cork ainsi qu'à Dublin, entre 1999 et 2001, dans le cadre d'une recherche doctorale consacrée aux usages sociaux des généalogies irlandaises. Cette étude, financée par la fondation Cetelem, le laboratoire d'Anthropologie sociale de l'École des hautes études en sciences sociales et l'Ireland Fund, est placée sous la direction de Françoise Zonabend (EHESS-LAS).

6. Le thème même du retour apparaît de manière récurrente dans la littérature irlandoaméricaine. Voir McKenZIE, 1998, et BINCHY, 1989.

7. GMELCH, 1983, p. 53.

8. Dumortier, 1992, de même que O'Connor et Cronin, éd., 1993, en mentionnent l'existence. 


\section{DESCENDANTS DE MIGRANTS ET GÉNÉALOGIE}

Intéressons-nous de plus près aux modalités mêmes de ces « retours » lorsqu'ils sont effectués dans le but de collecter des informations généalogiques, ainsi qu'à leurs acteurs principaux : les descendants de migrants ${ }^{9}$. Les indications fournies en 1994 par le ministère du Tourisme irlandais permettent de dresser une rapide cartographie de ces visiteurs bien particuliers, dont le nombre est estimé à 58000 pour l'année 1993 - soit 19000 de plus qu'en $1989^{10}$. Ces données quantitatives nous procurent également quelques estimations concernant leurs séjours. Âgés de vingt-cinq à quarante-cinq ans, ces amateurs d'histoire familiale viennent le plus souvent en Irlande accompagnés de leurs maris ou de leurs épouses. Venant en majorité d'Amérique du Nord (57\%) - Canada et États-Unis - durant la période estivale $(47 \%)$, ils restent pour une dizaine de jours en Irlande $(51 \%)$ - principalement à Dublin $(56 \%)$ et dans le sud-ouest de l'île $(61 \%)$ - et y dépensent beaucoup de devises ${ }^{11}$. Pour aussi sommaires et grossières qu'elles soient, ces données mettent surtout en avant la catégorisation par le ministère du Tourisme irlandais d'un groupe de visiteurs particuliers que sont les « historiens de famille ». Et si elles rendent possible une comparaison des déplacements effectués par ces mêmes personnes avec ceux accomplis par d'autres - «golfeurs », « amateurs de sites archéologiques », etc. -, elles ne nous permettent pas en revanche d'appréhender ni de décrire de manière précise les pratiques de circulation qui leur sont propres.

Visites de cimetières, consultations de registres civils et paroissiaux, discussions avec des généalogistes professionnels ou amateurs sont les démarches bien connues auxquelles s'adonnent, de façon générale, les amateurs d'histoire familiale. Sillonnant le pays de bibliothèques en archives, de paroisses

9. De ce fait, je m'intéresserai ici davantage aux descendants de migrants qui effectuent des recherches généalogiques qu'aux autochtones qui les conduisent aussi. À ce propos, il est important de préciser que le discours indigène assimile souvent « historiens de famille » et descendants de migrants, à l'instar de cet autochtone qui me disait : «Les Irlandais n'ont pas besoin de faire de telles recherches car ils savent d'où ils viennent. Ils savent que depuis longtemps leurs ancêtres sont Irlandais et qu'ils sont nés dans ce pays. Ils savent aussi que ces derniers étaient fermiers. Leurs origines sont là. Pour les Américains et Australiens c'est différent. Il s'agit de nations nouvelles dont les habitants sont presque tous des descendants de migrants et la plupart ignore de quel pays viennent leurs ancêtres. » Pourtant la part des Irlandais engagés dans de telles recherches est loin d'être négligeable. N'oublions pas, en effet, que la professionnalisation de cette pratique a été instituée à la fin des années 1980 et qu'un cursus universitaire destiné à former les amateurs a également été créé. De même, et en dépit de leur non-visibilité, on compte de nombreux amateurs irlandais membres d'associations de généalogistes.

10. Il n'existe pas sur ce point précis de données plus récentes. Notons, cependant, que les personnes ayant rédigé le rapport de 1993 prévoyaient que ce nombre s'élèverait à 107000 en 1999.

11. Perspective on Irish tourism activities, 1989-1993, 1994. 
en espaces associatifs, les généalogistes - qu'ils soient autochtones ou non espèrent ainsi trouver des indications qui, une fois sélectionnées et manipulées, leur fourniront les clés de leur identité ${ }^{12}$. Mais il reste une autre pratique qui permet aux généalogistes d'enrichir leur savoir familial et de se trouver de nouveaux référents identitaires : les rassemblements claniques. L'expression « rassemblement clanique » est la traduction française de la formule anglaise «clan gathering» ou «clan rallye ». Également appelés « get-together meetings », ces événements visent à réunir des personnes portant un patronyme identique sur le lieu d'où ce nom de famille est originaire ${ }^{13}$. Leur développement, qui va de pair avec celui de la généalogie, est particulièrement fulgurant depuis la fin des années 1980. Ces manifestations, qui se déroulent le plus souvent en période estivale, sont l'occasion pour les participants venus du monde entier de se rencontrer ${ }^{14}$. Durant les deux ou trois jours que durent ces réunions, des ateliers de discussion sont proposés autour des thèmes de famille et d'émigration. Des visites sont organisées afin de faire découvrir aux participants les lieux où vivaient leurs ancêtres les plus lointains - c'est-à-dire les premiers porteurs de leurs patronymes mais aussi de toutes les personnalités locales ou internationales qui les ont par la suite portés -, et des veillées folkloriques leur sont proposées avec danses et chants traditionnels ${ }^{15}$.

À travers l'ensemble des démarches auxquelles ils prennent part, ces visiteurs particuliers que sont les « historiens de famille » tentent tout d'abord d'identifier et de commémorer leurs morts. Que ce soit en lisant le nom des défunts sur des registres civils ou paroissiaux, en racontant leurs vies, ou en se rendant sur les lieux que ces derniers occupaient, les généalogistes cherchent en effet à raviver leurs souvenirs et à se construire une histoire familiale. En cela, leur quête, qu'elle soit mémorielle et/ou identitaire, témoigne de l'attachement qu'ont ces vivants envers le passé et envers les espaces qui lui sont attachés ${ }^{16}$. Le discours

12. De nombreuses recherches ont récemment été conduites sur ce sujet. Voir, notamment, les articles de Segalen et Michelat, 1991, de Sagnes, 1995, ainsi que Barthélemy et PINGAUD, dir., 1997.

13. Ces pratiques ne sont pas spécifiques à l'Irlande. SAgnes, 1998, rapporte que de tels événements, qu'elle désigne sous le terme de «cousinade », se déroulent aussi dans le Languedoc.

14. Le nombre de personnes qui prennent part à de telles réunions est variable, et dépend en partie de la médiatisation de ces événements. Le rassemblement O'Mahony qui s'est déroulé en juin 1999 dans le comté de Cork réunissait une cinquantaine de participants, contre près de 1000 pour celui organisé pour les O’Donovan en juin 2000 et auquel a pris part le ministre de la Culture, Síle de Valera.

15. Le choix de ces chants n'est pas laissé au hasard. Leurs paroles évoquent la Famine - Revenge for Skibbereen -, l'émigration - My bonne is over the ocean - ou encore la nostalgie de leurs auteurs envers certains lieux - Bantry Bay, On the banks at my own lovely Lee.

16. LEGRAND, à paraître. 
de l'un de mes informateurs lors de l'inauguration d'un rassemblement clanique le montre bien :

«Castle Donovan, c'est notre lieu d'origine, notre foyer ancestral. Et ce foyer («home»), c'est le lieu d'appartenance de la famille. 'Chez nous' a une merveilleuse résonance. Aucun voyage n'est trop long tant qu'il nous ramène chez nous. Nos ancêtres ont disparu, et pourtant à travers les liens de sang, ce sont nos ancêtres qui nous ont appelés ici aujourd'hui. Nous honorons tous les O’Donovan décédés, nous chérissons tous les O’Donovan vivants aujourd'hui, et nous comptons sur les jeunes O'Donovan pour l'avenir. »

Car la commémoration n'est pas seulement le fait d'individus isolés. Des groupes entiers de personnes venues en Irlande pour découvrir leurs « racines » peuvent en effet prendre part à des célébrations religieuses. Prières, messes, bénédictions sont d'ailleurs généralement inscrites à l'ordre du jour des rassemblements claniques, afin que ne soient pas oubliés ceux qui sont morts pendant la Grande Famine ou dans les «bateaux-cercueils » à destination du Nouveau Monde.

Au-delà de leur aspect commémoratif, ces voyages effectués sur le sol irlandais par des descendants de migrants ont aussi une portée sociale. Dans un ouvrage collectif intitulé Milieux et mémoire, l'anthropologue Marc Augé mentionnait que :

« La mémoire symbolique a [...] besoin de la mémoire rituelle pour devenir sociale. La commémoration, à l'inverse du souvenir, appartient à tous. Elle procède d'une volonté explicite de solidarité. C'est cette volonté de solidarité que partagent d'abord ceux qui commémorent et qui n'ont pas tous le même rapport individuel au passé qu'ils commémorent ${ }^{17}$. »

En effet, lorsqu'ils sont ritualisés, les retours des descendants de migrants participent à la construction d'une sociabilité. Ils leur permettent en effet de rencontrer des autochtones, de partager un tant soit peu leurs modes de vie, et d'échanger avec eux des informations relatives à leurs histoires de vie respectives ou à celles de leurs aïeuls. Par les contacts qu'ils établissent en Irlande, les généalogistes peuvent aussi obtenir des indications concernant les réseaux ou les outils à mobiliser pour mener à bien leurs investigations. Les rassemblements claniques sont particulièrement propices à l'établissement de réseaux d'entraide entre autochtones et résidents étrangers. Constituant de véritables espaces d'interactions, ils créent du lien social entre les participants plus qu'ils ne leur certifient l'existence d'interconnexions familiales supposées. L'un de mes

17. Augé, 1993, p. 23. 
interlocuteurs irlando-américain à qui j' avais demandé ce qu'il attendait d'une telle manifestation m'avait répondu : «Je ne sais pas trop. Celle-ci revêt un aspect éducatif aussi bien que social. Je ne m'attends pas à y rencontrer des proches parents. Je ne pense pas en trouver ici. » En effet, les réunions claniques donnent souvent lieu à divers échanges : de cartes de visite surtout, mais aussi de services. « Ça serait bien que tu ailles à Orléans voir ce que sont devenus les ..., ceux qui ont participé au rallye clanique dans les années 50 », me dit l'un des organisateurs d'un rallye clanique auquel j'ai participé. « Il faudrait également que tu te renseignes sur la provenance et la signification du patronyme que porte l'une de mes arrière-grand-mères maternelles. Est-ce que c'est français ? "De", la particule, c'est typiquement français n'est-ce pas ? », enchérit un Anglais dont le regard trahissait l'espoir que je lui apporte la confirmation immédiate de ce que ses intenses recherches archivistiques n'avaient pas pu prouver, c'est-à-dire pour reprendre une expression de l'anthropologue Robin Fox : « [qu'il n’était pas] tombé du ciel, sans passé, sans histoire... [et qu'il] avait des "racines"18. »

Ces voyages effectués par des généalogistes descendants de migrants irlandais ont enfin une visée didactique. Ils sont en effet pour eux tout autant l'occasion de visiter l'Irlande, de rencontrer ses habitants, que de découvrir des indications biographiques concernant leurs ancêtres proches ${ }^{19}$. Ils leur permettent aussi de découvrir - ou d'en apprendre davantage sur - l'histoire mythique - l'arrivée des Celtes sur l'île -, ancienne - la période prénormande - et plus récente - la Grande Famine et l'émigration - de ce pays. Toutes ces informations, fortement chargées sur le plan symbolique, fournissent des référents identitaires aux visiteurs, mais aussi à leur descendance. Un de mes informateurs irlandoaméricain, venu en Irlande avec neuf autres membres de sa famille m'expliquait : «Je veux, ma femme et moi nous voulons, que nos fils comprennent que c'est grâce à ce que nos ancêtres ont enduré, grâce à leurs sacrifices, que notre niveau de vie est aujourd'hui élevé. »

\section{LES USAGES POLITIQUES D'UNE DEMANDE IDENTITAIRE}

Processus de reterritorialisation identitaire pour les descendants de migrants irlandais, les visites que ces derniers effectuent en Irlande pour y découvrir des liens de parenté ont aussi pour le gouvernement irlandais une visée économique et sociale. En se servant notamment de la généalogie pour créer des emplois

18. Fox, 1967, p. 33.

19. Le manque d'archives civiles ou paroissiales, leur non-suivi, ainsi que la disparition de certaines d'entre elles durant la guerre civile constituent autant d'obstacles pour les généalogistes. 
dans le secteur tertiaire et pour attirer sur l'Île des milliers de touristes en quête d'identité, l'Irlande semble ainsi, de par sa politique volontariste, se démarquer fortement de ces partenaires européens ${ }^{20}$.

En 1984 fut fondée dans le comté de Kildare The Irish Family History Society. Ses fondateurs - des historiens amateurs - étaient désireux de se voir faciliter l'accès des généalogistes aux sources écrites, tout en minimisant le risque de détérioration qu'ils pouvaient causer à ces dernières. L'originalité de cette association résidait dans les moyens qu'elle allait employer pour parvenir à ses fins. Sa volonté d'informatiser l'ensemble des documents archivistiques était nouveau en Irlande, et le fait que l'intégralité de ces matériaux puissent être un jour accessibles à travers un support informatique devait révolutionner la pratique généalogique. Un programme d'action permettant la mise en place de ce processus fut créé en 1987. Étendu à l'Irlande du Nord dès 1989, ce projet prit le nom de The Irish Genealogical Project (IGP). Sa réalisation devait passer par l'établissement à travers le pays de plusieurs structures d'accueil à l'intérieur desquelles les généalogistes amateurs auraient la possibilité de consulter des bases de données informatiques et d'être aiguillés dans leurs démarches par des professionnels. L'une des premières étapes de cette entreprise fut donc l'indexation informatique des documents archivistiques - actes civils de naissance, de mariage et de décès ; actes paroissiaux de baptêmes, de mariages et de mise en sépulture ; recensements démographiques, etc. De nombreux sites de recherche furent depuis créés - le premier d'entre eux ayant ouvert ses portes à Ennisco, dans le comté de Mayo, en avril 1992.

Si les motivations initiales des fondateurs étaient d'ordre utilitaire, elles furent rapidement utilisées à des fins politiques et économiques. Dès 1990, l'un des responsables de l'IGP redéfinissait d'ailleurs les buts de cette entreprise en ces termes : «Le but de l'IGP est d'atteindre la diaspora irlandaise et de faire en sorte que des personnes descendants d'Irlandais viennent en Irlande, passer de leur temps, dépenser leur énergie, et espérons-le, un peu de leur argent. Pour simplifier, c'est lié au tourisme ${ }^{21}$. »

Il s'agissait donc pour les organisateurs et mécènes de ce programme, de faire en sorte que des milliers de descendants de migrants irlandais viennent en Irlande pour tenter d'y découvrir des informations biographiques concernant leurs ancêtres et pour essayer d'y renouer des liens avec de lointains parents, et surtout de tirer un bénéfice financier des séjours de ces visiteurs. Car, faut-il le rappeler, l'Irlande fut touchée dès le début des années 1980 par une période de récession économique, et l'une des stratégies politiques mises en place pour y

20. L'anthropologue Joël Candau a montré qu'en France certaines sociétés tirent profit de l'engouement actuel pour les généalogies, voir BARTHÉLEMY et PINGAUD, dir., 1997, p. 119-129. Mais, contrairement à ce qui se passe en Irlande, le développement de ce marché ne semble pas, en France, mû par une quelconque volonté politique.

21. Tunney, 1990, p. 61 
faire face était de développer l'activité touristique dans ce pays, afin d'en diversifier les ressources jusque-là issues principalement du secteur agricole ${ }^{22}$. Des projets destinés notamment à faire découvrir aux touristes l'histoire de l'Irlande et les modes de vie des populations autochtones furent élaborés, et des musées créés. Parmi ces programmes qui reçurent l'aval du Premier ministre (The Department of the Taoiseach) et des fonds du ministère du Tourisme irlandais (The Bord Fáilte) figure l'IGP. Dès septembre 1985, le gouvernement avait en effet remarqué que :

«On a estimé à plus de 50 millions le nombre de personnes descendant d'Irlandais et vivant à l'étranger. C'est un potentiel énorme pour ce qu'on pourrait appeler le tourisme mémoriel. Cela nécessite des efforts soutenus pour son développement. Bien que les gens d'ascendance irlandaise aient quelques prédispositions pour visiter l'Irlande, l'expérience nous a montré que des efforts considérables en marketing sont nécessaires pour convertir ce capital en réel séjour ici ${ }^{23}$. »

Et l'on comprend mieux dès lors pourquoi le ministère du Tourisme irlandais participe, à partir de cette époque, à la promotion des rassemblements claniques en publiant, dans l'un des journaux ${ }^{24}$ qu'il produit, des indications concernant la tenue prochaine de ces événements, et un manuel destiné aux généalogistes. Intitulée « Tracing your Irish ancestors » et diffusée à diverses associations de généalogistes amateurs et professionnels, cette notice émet le conseil suivant :

« Une fois que vous avez trouvé vos racines sur le papier, la phase suivante est le retour au pays («Homecoming »). Non seulement vous devriez y vérifier l'emplacement de votre lieu d'origine, mais vous devriez aussi explorer ses alentours ainsi que les villages et les villes avoisinants. De cette manière, vous pourrez vous imprégner et savourer la culture et l'histoire de cette région tout entière ${ }^{25}$. »

D'autres partenaires sont également impliqués dans ce projet ${ }^{26}$, comme le Fonds international pour l'Irlande (International Fund for Ireland). L'IGP

22. Voir, à ce sujet, DeEgan et Donal, 1993, ou encore Kockel, éd., 1994.

23. The Government, 1985, p. 27.

24. Cette revue semestrielle intitulée Ireland of the Welcomes est éditée depuis 1952. Dans l'une de ses rubriques intitulée « Shopping your Irish Treasure », des annonces faites par certains généalogistes professionnels en quête de clientèle sont publiées ainsi que celles de certaines boutiques spécialisées dans la vente de matériaux utiles aux amateurs de cette pratique.

25. Tracing your ancestors in Ireland, s. d., p. 16.

26. Je ne mentionnerai ici que les principaux organismes nationaux et internationaux qui ont participé à la réalisation de ce projet et renverrai à Comptroller and Auditor General, 1996, pour une liste complète des mécènes et des sommes investies par chacun d'eux. Dans ce rapport, il est précisé que près de 15 millions de livres irlandaises auraient été engagées entre 1988 et 1996 dans l'IGP (p. iii). Síle de Valera a renouvelé le financement de ce projet pour l'année 1999-2000. 
constituant l'un des nombreux programmes de coopération économique entre le Nord de l'Île et la République, le mécénat de cet organisme fut destiné à ce titre à encourager le dialogue entre ces deux parties et à favoriser leur réconciliation ${ }^{27}$. Mary Robinson, présidente de la république d'Irlande de 1990 à 1997, précisa d'ailleurs, dans un discours qu'elle adressa au parlement en février 1995, que :

« Avec le Projet Généalogique Irlandais, soutenu par les deux gouvernements, on peut transférer actuellement vers l'ordinateur les transcriptions manuscrites des registres locaux de naissances, de morts et de mariages. On se sert de la technologie moderne pour permettre aux hommes et aux femmes, dont les origines sont recensées dans des registres de Kerry à Antrim, d'y avoir accès. Ce faisant, le Projet fournit de l'emploi et de la formation pour les jeunes gens dans les domaines de la technologie et de l'histoire. Par ailleurs, l'établissement récent d'un conseil d'organisations généalogiques, qui implique de nouveau les deux parties de cette île, démontre le potentiel d'une coopération volontaire ${ }^{28}$. »

Comme l'a montré Vincent Hernot dans un article consacré à « La diaspora dans les discours de Mary Robinson », celle-ci voit notamment dans la migration de milliers d'Irlandais « ce qui rassemble les deux communautés [irlandaise et nord-irlandaise], et par conséquent elle essaie de s'en servir comme dénominateur commun qui les rapprocherait ${ }^{29} »$. L'IGP constitue à ce titre, pour l'ancienne présidente de la République comme pour le gouvernement de son pays d'ailleurs, non seulement un moyen de réunir les Irlandais de la République de ceux d'ailleurs ${ }^{30}$, mais aussi l'une des manières concrètes de parvenir à une coopération économique entre l'Irlande du Nord et du Sud.

The Foras Áiseanna Saothair (FÁS) a également largement contribué à la réalisation de l'IGP en fournissant, en tant que centre d'aide à la formation et à l'insertion professionnelle, la main-d'œuvre nécessaire à l'indexation informatique des sources archivistiques. De nombreux jeunes autochtones ont pu de ce fait bénéficier de ce programme pour recevoir une qualification d'opérateur de saisie au terme des six mois à temps plein que dure leur contrat de formation.

Ainsi, le déploiement de tout un réseau d'infrastructures destiné à ces descendants de migrants en quête de savoir généalogique est l'un des résultats des nouvelles orientations politiques dessinées en Irlande dans les années 1980. Mais il témoigne aussi de mutations récentes dans la manière de s'imaginer et de représenter l'identité irlandaise.

27. Pour plus de détails sur les financements accordés par le Fonds international pour l'Irlande, je renvoie à l'article de BEVANT, 1994.

28. ROBINSON, 1995.

29. Gallagher, dir., 1998, p. 84.

30. Le discours autochtone distingue les Irlandais d'ici «The Irish at home » et ceux d'ailleurs «The Irish abroad », cette dernière expression étant utilisée de manière générique pour désigner les migrants irlandais et leurs descendants. 
RETOUR AU PAYS ANCESTRAL

ET REPRÉSENTATIONS DE L'IDENTITÉ IRLANDAISE

Qu'elle soit pensée par les autochtones, imaginée et encouragée par les politiciens ou réalisée par les descendants de migrants, cette notion même de « retour » pose, enfin, la question des représentations que se font ces personnes de l'identité irlandaise.

La première est celle des représentations que se font aujourd'hui ces personnes de l'émigration. Car pour que l'on puisse aujourd'hui parler de retour, il faut qu'il y ait eu au préalable un départ. Si celui-ci est bien souvent le fait d'un de ses aïeux, la manière dont un individu perçoit un tel déplacement modèle l'image qu'il a de lui-même et de son groupe familial. Or, il apparaît que l'émigration irlandaise vers d'autres mondes constitue pour mes informateurs bien plus qu'un simple mouvement de population. En effet, elle est considérée plus comme un exil que comme une migration. Le premier terme à la différence du second est perçu comme un acte subi, imposé, non désiré et pensé comme une fatalité. «Ils n'avaient pas d'autre choix que de partir. C'était partir ou mourir», m'expliquèrent certains de mes informateurs à propos des conditions dans lesquels leurs aïeuls ont quitté l'Irlande. La réduction fréquente par les « historiens de familles » amateurs et par certains politiciens - Robinson en particulier - des causes de la migration à la Grande Famine en renforce d'ailleurs le caractère tragique. En cela, l'émigration irlandaise se rapproche dans les discours de l'exode et de la diaspora ${ }^{31}$. Cet exil, qui constitue selon les historiens et les philosophes ${ }^{32}$ une des données essentielles de l'Irlande moderne, représenterait aussi pour les descendants de migrants établis aux États-Unis, une caractéristique qui les distinguerait d'autres groupes de populations immigrées. Ainsi, le fait que des millions d'Irlandais aient été contraints à quitter le pays renforce chez leurs descendants le sentiment d'appartenir à une population exclue de son milieu d'origine.

Par ailleurs, de par l'importance en nombre de ceux qui peuvent aujourd'hui revendiquer une ascendance irlandaise, l'Irlande se trouve assimilée à un berceau des civilisations américaines et océaniennes. Qualifiée par certains de « nurserie de migrants ${ }^{33} »$, le discours populaire veut aussi que l'Irlande ait apporté à d'autres pays une main-d'œuvre qualifiée et un savoir-faire. Lors d'un rassemblement clanique, il avait été dit aux participants : «D'où les Américains tiennent-ils leurs savoirs ? Comment ont-ils appris à construire les villes ? », et la réponse implicite était : «Des descendants de migrants ». Le fait que les

31. L'émigration irlandaise est d'ailleurs, depuis plusieurs siècles déjà, comparée à la diaspora juive. Voir notamment, au sujet de cette assimilation, le récent ouvrage de LACOUELABARTHE et GOLDRING, 2001.

32. Notamment chez KeARnEY, 1997.

33. Voir, notamment, MaC LaUGHLin, 1994. 
migrants irlandais aient activement participé à la construction tant politique que sociale des États-Unis était récurrent dans ce discours, et contribuait à élaborer une image positive des Irlandais auxquels diverses vertus étaient attribuées. Ils étaient dits « organisateurs », « aventuriers », avec un «goût prononcé pour le travail collectif ». L'évocation de nombreuses personnalités d'origine irlandaise résidant - ou ayant vécu - ailleurs qu'en Irlande, participait également à ce processus de mise en avant de la réussite sociale du migrant et de sa descendance. En évoquant oralement ou en mentionnant dans leurs écrits le succès de leurs proches, ces généalogistes expriment ainsi leurs fiertés de se sentir ou de se définir comme Irlandais ${ }^{34}$. Il en était de même lorsque ces derniers faisaient aussi allusion aux «difficiles conditions de vie » dans lesquelles se trouvaient leurs aïeuls, tant sur le sol irlandais que dans le Nouveau Monde. La réutilisation par ces « historiens de familles » de qualificatifs autrefois employés par les nativistes américains pour dépeindre leurs ancêtres installés outreAtlantique - « analphabètes, sales, pauvres et alcooliques »-y était en effet tout aussi fréquente. Autant d'adjectifs, qui servaient dans ce contexte à rappeler que les migrants et leurs descendants avaient réussi à surmonter les obstacles de l'immigration et qu'ils s'étaient insérés tout en conservant comme spécificités une solidarité intercommunautaire et un attachement à leurs origines irlandaises.

Autrefois terre d'émigration, l'Irlande est en effet aujourd'hui présentée dans les brochures touristiques, mais aussi par ses politiciens, comme une terre d'accueil. « Nous, en Irlande, adorons accueillir les personnes qui sont de retour », déclarait la ministre de la Culture, Síle de Valera. Certains autochtones l'affirment aussi, mais de manière plus nuancée : «Je dirais que les personnes en Irlande sont souvent aimables, accueillantes et sociables [...] Venez nous rendre visite... mais ne restez pas trop longtemps ! » Par son hospitalité affichée, l'Irlande semble ainsi offrir à ceux qui sont en quête d'identité leur rattachement à une histoire collective qui se veut ancienne et héroïque. Cette ancienneté du peuple irlandais est en effet racontée aux participants des rallyes claniques à travers l'évocation des exploits des Gaels - leur conquête de l'île, leur résistance aux Vikings et aux Normands. De même, le fait que l'Irlande dispose non seulement de nombreux vestiges archéologiques mais aussi d'un des corpus généalogiques les plus anciens d'Europe est souvent mis en avant dans les discours des amateurs ou même dans celui des personnages politiques. Il apparaît aussi, au regard des entretiens menés auprès de généalogistes amateurs américains et australiens, que leur volonté d'inscrire leurs histoire dans un cadre qui dépasserait celui de leurs nations est grande ${ }^{35}$. C'est le cas entre autres de ce couple d'Irlando-Américains de trente ans, de passage à la bibliothèque nationale de

34. Je renvoie à l'article de BYRON, 1998, sur le sentiment d'appartenance à la « culture irlandaise ».

35. Voir notamment, sur ce sujet, les ouvrages de Collard, 1999, sur le Canada et celui de Le Menestrel, 1999, sur la Louisiane. 
Dublin, et qui espéraient pouvoir se raccrocher à cette mémoire longue de l'histoire irlandaise. « Nous venons chercher ici l'histoire que les États-Unis ne peuvent nous fournir en tant que nouveau pays », énonçaient-ils.

La réactivation des liens entre les Irlandais de «la République » et « ceux d'ailleurs » pose enfin la question de la temporalité de ces mouvements de circulation et de la filiation d'une identité irlandaise. En effet, le « retour » en Irlande de ces descendants de migrants permet de penser différemment encore la migration. À cette occasion, elle retrouve le caractère provisoire qui lui était parfois initialement conféré par les partants. En « revenant» en Irlande, leurs descendants instaurent un semblant de continuité temporelle qu'ils réaffirment à travers les récits généalogiques qu'ils produisent. Ceux-ci y mettent ainsi en avant la figure du migrant et celle de leurs parents, mais accordent une moindre prégnance à celles qui peuplent les générations intermédiaires. De ce fait, les généalogistes se représentent l'identité irlandaise comme immuable en dépit des ruptures provoquées par les migrations. Il en va de même des discours énoncés par les politiques. «Vous êtes venus ici pour revendiquer ce qui est une partie très partagée de votre patrimoine [...] et pour célébrer cette filiation, ce lien et cette continuité ", disait d'ailleurs Valera, lors de sa venue à un rallye clanique. L'identité irlandaise est aussi de ce fait posée comme ne pouvant être restreinte à un cadre strictement territorial. Elle dépasserait non seulement les frontières nationales - que sont celles entre l'Irlande du Nord et la République - mais aussi les limites géographiques de l'île - l'Irlande, comme trait d'union entre l'Ancien Monde et le Nouveau -, comme le soulignent notamment Robinson ${ }^{36}$ et plus récemment son successeur à la présidence de la République, Mary McAleese ${ }^{37}$.

Les retours des descendants de migrants sur le sol irlandais semblent ainsi témoigner d'un double réagencement identitaire. Lorsqu'ils sont effectués dans le but de découvrir des indications biographiques, ces voyages permettent à l'individu de se construire une histoire et une identité familiales. Mais ils sont aussi pour eux l'occasion de revendiquer une identité irlandaise dont nous avons montré qu'elle faisait elle-même l'objet de mutations et de manipulations. Par ailleurs, ces déplacements, qui donnent lieu à la création de nouveaux espaces d'interaction entre les Irlandais d'ici - de la République - et ceux d'ailleurs, s'inscrivent dans une dynamique de la circulation touristique d'une part et

36. Après avoir déclaré lors de son investiture à la présidence de la République, que près de 70 millions de personnes pouvaient à travers le monde revendiquer une ascendance irlandaise, RoBInson, 1993, p. 8, expliqua lors de l'ouverture du premier congrès irlandais de Généalogie, que ni l'expérience irlandaise ni l'expression culturelle de «l'irlandité » ne peuvent être confinées à l'espace insulaire.

37. L'actuelle présidente de la République, Mary MCAlEESE, citée dans The Irish Post du $1^{\text {er }}$ janvier 1997, déclarait à son tour en 1997 « qu'être irlandais signifie plus que de vivre sur l'île d'Irlande ». 
dans une logique politico-économique de l'autre. Leur étude permet, enfin, de montrer comment une société - en l'occurrence l'Irlande - peut participer directement à la sélection des individus qu'elle entend accueillir pour un temps limité et dont elle espère tirer bénéfice.

Caroline Legrand

(septembre 2001). 


\section{LISTE DES RÉFÉRENCES}

Augé (Marc), 1993, « Ouverture scientifique », in Alvarez-Pereyre (Frank), éd., Milieux et mémoire, Jérusalem, Centre de recherche français de Jérusalem, p. 13-25.

BARThÉlemy (Tiphaine) et PINGAUd (Marie-Claude), dir., 1997, La Généalogie entre sciences et passion, actes du CXX $\mathrm{CX}^{\mathrm{e}}$ congrès des Sociétés historiques et scientifiques, Aix-en-Provence, 24-26 oct. 1994, Paris, Éd. du Comité des travaux historiques et scientifiques/Section anthropologie et ethnologie françaises.

Bevant (Yann), 1994, «Le Fonds international pour l'Irlande et la Communauté européenne », Études irlandaises, ${ }^{\circ}$ hors-série « L'Irlande aujourd'hui », p. 155-170.

Binchy (Maeve), 1989, Retour en Irlande, trad. Ives et Cécile Trévian, Paris, Presses de la Cité.

BYRON (Reginald), 1998, «Ethnicity and generation. On "Feeling Irish" in contemporary America », Ethnologia Europaea, 28, p. 27-36.

Collard (Chantal), 1999, Une famille, un village, une nation. La parenté dans Charlevoix, 1900-1960, Montréal, Les Éditions du Boréal.

Comptroller and Auditor General, 1996, The Irish Genealogical Project, Dublin, Stationery Office Publications (Report on Value for Money Examination, 14).

DeEgan (James) et Donal (Dineen), 1993, « Irish tourism policy. Targets, outcomes and environmental considerations », in O'CONNOR (Barbara) et CRONIN (Michael), éd., Tourism in Ireland. A critical analysis, Cork, Cork University Press, p. 1-10.

DumORTIER (Brigitte), 1992, «Les clés de l'essor touristique irlandais », Hommes et terres du Nord, 2, p. 79-85.

DUPÂQUIER (Jacques), 1980, «Les aventures démographiques de la France et de l'Irlande (XVIII ${ }^{\mathrm{e}}-\mathrm{XX}^{\mathrm{e}}$ siècles) », in CULLEN (Louis M.) et FuRET (François), dir., Irlande et France, XVII $-X X^{e}$ siècles. Pour une histoire rurale comparée, actes du $\mathrm{I}^{\mathrm{er}}$ colloque franco-irlandais d'Histoire économique et sociale, Dublin, 1977, Paris, Éd. de l'École des hautes études en sciences sociales, p. 167-180.

Fox (Robin), 1967, Anthropologie de la parenté. Une analyse de la consanguinité et de l'alliance, Paris, Gallimard.

Gallagher (Monique), dir., 1998, Irlande-Exils, actes du colloque de la Société française d'études irlandaises, Nice, 20-21 mars 1998, Cycnos, vol. XV, 2.

GMELCH (George), 1983, "Who returns and why ? Return migration behaviour in two North Atlantic societies », Human Organization, vol. XLII, 1, p. 46-54.

Hannan (Damian), 1979, Displacement and development. Class, kinship and social change in Irish rural communities, Dublin, The Economic and Social Research Institute (General Research Series, 96).

Kearney (Richard), 1997, Postnationalism Ireland. Politics, culture and philosophy, Londres, Routledge.

KocKel (Ullrich), éd., 1994, Culture, tourism and development, Liverpool, Liverpool University Press. 
KocKel (U.), éd., 1995, Landscape, heritage and culture, Liverpool, Liverpool University Press.

Lacoue-Labarthe (Isabelle) et Goldring (Maurice), 2001, Le Trèfle et l'étoile. Juifs et Irlandais : histoires parallèles, mémoires croisées, Paris, Éditions Autrement (Mémoires, 70).

LEGRAND (Caroline), à paraître, «De l'individuel au collectif. Pratiques et discours généalogiques en Irlande », in Représentations de soi. Mimésis et simulation, actes du colloque du Centre d'études et de recherches irlandaises de l'université Charles de Gaulle-Lille III, juin 2001, Villeneuve-d'Ascq, Presses universitaires du Septentrion.

Le Menestrel (Sara), 1999, La Voie des Cadiens. Tourisme et identité en Louisiane, Paris, Belin.

MACÉInRí (Piaras), 1997, «Some recent demographic developments in Ireland», Études irlandaises, vol. XXII, 1, p. 145-159.

MAC LAUghlin (Jim), 1994, Ireland. The emigrant nursery and the world economy, Cork, Cork University Press (Undercurrents, 8).

McKenZIE (Richard), 1998, Turn left at the Black Cow. One family's journey from Beverly Hills to Ireland, New York, Rinehart ed.

O'Connor (Barbara) et Cronin (Michael), éd., 1993, Tourism in Ireland. A critical analysis, Cork, Cork University Press.

O'Toole (Fintan), 1998, The Lie of the land. Irish identities, Dublin, New Island Books.

Perspective on Irish tourism activities, 1989-1993, 1994, Dublin, Bord Fáilte Publications.

RoBInson (Mary), 1993, « Adress by an tUachtarán, Mary Robinson », in Aspects of Irish genealogy, proceedings of the First Irish genealogical congress, sept. 1991, éd. Irish Genealogical Congress Committee, Naas, p. 7-8.

Robinson (M.), 1995, «Cherishing the Irish diaspora », Address to the Houses of the Oireachtas, 2 Febr.

Sagnes (Sylvie), 1995, « De la terre et du sang. La passion généalogique », Terrain, 2, p. 125-126.

SAGNES (S.), 1998, «Une parenté sur mesure... Les nouvelles formes de parenté à l'épreuve de l'acharnement généalogique », in FINE (Agnès), éd., Adoptions. Ethnologie des parentés choisies, Paris, Maison des sciences de l'homme (Ethnologie de la France, 19), p. 275-309.

Segalen (Martine) et Michelat (Claude), 1991, «L'amour de la généalogie », in SEgalen (M.), dir., Jeux de famille, Paris, Éditions du CNRS, p. 193-208.

The Government, 1985, White paper on tourism policy, Dublin, Stationery Office Publications.

Tracing your ancestors in Ireland, s. d., Dublin, Bord Fáilte Publications.

TunNEY (John), 1990, « The IGP. A tourist agenda for genealogy », Irish Family History. The Journal of the Irish Family Historical Society, vol. VI, p. 61-69.

Verrière (Jacques), 1979, La Population de l'Irlande, Paris, Mouton. 